\title{
MODELAGEM DE PLANOS DE RECOMPOSIÇÃO DE SISTEMAS ELÉTRICOS COMO GRAFOS CPM/PERT
}

\begin{abstract}
Alexandre de Assis Mota*
assis@dsee.fee.unicamp.br

\section{RESUMO \\ Esse trabalho formaliza um modelo de grafos CPM/PERT para viabilizar a representação de planos de recomposição de sistemas elétricos e expõe uma metodologia para deriva- ção automática do mesmo a partir da lista de atividades de um procedimento de recomposição. Essa técnica de mon- tagem automática foi aplicada com sucesso a uma instrução operativa de recomposição pós-blecautes do sistema interli- gado nacional (SIN), referente a uma porção da rede elétrica do sul do Brasil, que compreende as usinas hidrelétricas de Capivara e Taquaruçu, o corredor de transmissão associado a essas usinas e as subestações de Assis e Londrina.}

\author{
Lia Toledo Moreira Mota* \\ lia@dsee.fee.unicamp.br
}

\author{
André Luiz Morelato França* \\ morelato@dsee.fee.unicamp.br \\ ${ }^{*}$ DSEE/FEEC/UNICAMP - Avenida Albert Einstein, 400 - Cidade Universitária - Campinas (SP) - Brasil - C.P. 6101 - CEP \\ 13083-852, Fone: +55-0xx-19-37883708, Fax : +55-0xx-19-32891395
}

PALAVRAS-CHAVE: Blecautes, Métodos de Caminho Crítico, Programação Orientada a Objetos, Recomposição de Sistemas de Energia Elétrica.

\section{ABSTRACT}

This work proposes a computational model based on PERT/CPM graphs to represent power system restoration plans. This model can be automatically derived using the precedence list represented by the restoration actions. This method was successfully applied to a restoration plan devel-

\footnotetext{
Artigo submetido em 01/11/2005

1a. Revisão em 12/06/2006

2a. Revisão em 09/10/06

Aceito sob recomendação do Editor Associado

Prof. Carlos A. Castro
}

oped for a South-Brazilian power network, generating satisfactory results.

KEYWORDS: Blackouts, Critical Path Methods, Objectoriented programming, Power System Restoration.

\section{INTRODUÇÃO}

No contexto do planejamento da recomposição de sistemas de potência, torna-se bastante desejável a especificação de um modelo computacional para representar planos de recomposição que viabilize, na medida do possível, o tratamento dos problemas inerentes às atividades de reenergização, segundo uma abordagem lógico-matemática bem definida. Deve ser objetivada, na escolha dessa abordagem, a sua capacidade de representar a natureza bastante diversa das inúmeras tarefas que são executadas durante o processo de recomposição, envolvendo atividades de caráter técnico (como, por exemplo, a atuação em controles e a atenção aos limites dos equipamentos e linhas de transmissão), gerencial (como a coordenação de interligação de diferentes áreas geo-elétricas), logístico (como o deslocamento de viaturas e equipes em campo) e estratégico (como a representação de cargas prioritárias para reenergização) (Adibi, 2000). Mais ainda, dois aspectos muito importantes têm que ser levados em conta na definição de um modelo para os planos de recomposição: a possibilidade de qualificar as diferentes linhas de ação definidas para abordar a reenergização sob um mesmo cenário de falta de energia, contemplando os diferen- 
tes aspectos do processo, e a viabilidade para modelar o caráter impreciso das atividades de recomposição, permitindo uma abordagem estocástica na sua especificação.

Assim, esse trabalho propõe uma metodologia para construção de um modelo computacional para os planos de recomposição (Mota et. al., 2004), baseado em métodos de caminho crítico (usualmente definidos como CPM/PERT), para representar o seqüenciamento temporal e a dependência lógica entre as atividades de recomposição.

Os métodos de caminho crítico CPM ("Critical Path Method") e PERT ("Program Evaluation Review Technique") são métodos de planejamento e programação de processos desenvolvidos, durante a década de 1950, pela Marinha dos Estados Unidos e pela companhia DuPont, com o objetivo de otimizar os processos com múltiplas atividades encadeadas, tanto no aspecto temporal quanto de aplicação de recursos (Moder, 1970; Sivazlian, 1975). Esses métodos definem uma base formal e uma abordagem generalizada para o gerenciamento de projetos e abrangem as características desejadas de flexibilidade e tratamento de imprecisões, desejadas para o modelo de planos de recomposição. Ambas as técnicas presumem que qualquer projeto ou plano pode ser desmembrado em uma sequiência lógica de ações predefinidas (ou atividades) encadeadas. A realização dessas atividades conduz à conclusão do projeto. Outra hipótese importante adotada nesses métodos é que as atividades possuem uma execução lógica de natureza tecnológica, ou seja, apesar de poderem ser executadas de uma maneira relativamente independente, elas possuem, necessariamente, precedências tecnológicas entre si. Na sua formulação, assume-se que a relação entre duração e custos de cada tarefa do processo é bem conhecida, o que permite a estimação de diversos parâmetros de gerenciamento e desempenho, como a alocação ótima de recursos e o controle de custos do projeto.

\section{PLANOS DE RECOMPOSIÇÃO}

No Brasil, o produto gerado ao final da etapa de planejamento da recomposição é um conjunto de textos, denominados "instruções operativas de recomposição", que faz parte dos procedimentos de rede do Sistema Interligado Nacional (SIN), disponibilizados pelo Operador Nacional do Sistema Elétrico (ONS).

Assim, uma instrução operativa de recomposição, ou simplesmente instrução operativa (IO), é um documento que descreve as ações que devem ser tomadas e as condições que devem ser satisfeitas na operação do SIN, após um evento de interrupção de grandes proporções no fornecimento de energia elétrica.

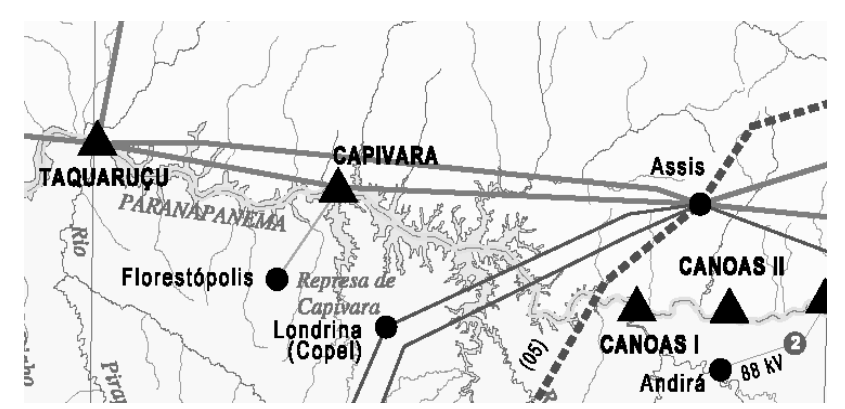

Figura 1: Área geo-elétrica Capivara.

\subsection{Formato Tabular de Instruções Ope- rativas}

É sugerida, pelo ONS, a representação das instruções operativas em um formato tabular, para facilitar o entendimento, pelos operadores e engenheiros, das atividades descritas no plano sob condições de recomposição. Esse formato torna explícitas características importantes do processo, tais como a ordem de uma determinada atividade na seqüência planejada de ações, o agente executor dessa atividade, os procedimentos específicos e as condições (ou controles) que devem ser atendidas antes que uma ação em questão possa se iniciar. Essas características ficam organizadas de uma maneira lógica, definindo o conjunto de procedimentos e ações em uma cadeia ordenada de atividades.

Como exemplo, a tabela I apresenta o conjunto de ações para a reenergização da área Capivara (área 16 da região Sudeste do Brasil), representada geograficamente na figura 1, que é formada pelas Usinas Capivara e Taquaruçu, pelas subestações de Assis e Londrina e pelo tronco de transmissão associado, extraído da instrução operativa correspondente (ONS, 2004).

\subsection{Lista de Ações de Recomposição}

No Brasil, como as ações estão delineadas em uma seqüência lógica nesse formato tabular, é possível determinar listas de atividades para cada instrução operativa, conforme detalhado, anteriormente, na análise geral de planos de recomposição. Essas listas devem explicitar a relação de precedência entre as diferentes ações de recomposição. A tabela II apresenta uma possível lista de atividades correspondente às ações seqüenciais de reenergização dos diferentes equipamentos elétricos da área Capivara, extraídas por inspeção da tabela I. Cada linha da tabela II identifica uma atividade com uma letra (A, B, C, etc.), apresenta brevemente a descrição da mesma e especifica suas atividades precedentes, ou seja, as ações que devem ser completadas para que a atividade de recomposição em questão possa se iniciar. 
Tabela I - Exemplo de Instrução Operativa - Área Capivara

\begin{tabular}{|c|c|c|}
\hline EXECUTOR & PROCEDIMENTO/AÇÃO & $\begin{array}{l}\text { CONDIÇÃO/ } \\
\text { CONTROLE }\end{array}$ \\
\hline Usina Capivara & $\begin{array}{l}\text { •Sincronizar } 2 \text { unidades gera- } \\
\text { doras. } \\
\text { •Energizar o TR-5 440/138 } \\
\mathrm{kV} \text { assumindo carga da re- } \\
\text { gião. } \\
\text { •Enviar tensão para Taqua- } \\
\text { ruçu. } \\
\text { •Confi rmar com o operador } \\
\text { de Assis se o reator RE-2 está } \\
\text { conectado no terminal da LT } \\
\text { e enviar tensão pela LT Capi- } \\
\text { vara / Assis. } \\
\text { •Quando da sincronização da } \\
3^{a} \text { unidade geradora, avisar o } \\
\text { operador de Assis. }\end{array}$ & $\begin{array}{l}\text { •Valor Máximo } \\
\text { de carga igual a } \\
120 \mathrm{MW} \\
\text {-Tensão em } \\
\text { torno de } 420 \mathrm{kV} \text {. } \\
\text {-Tensão } \leq 420 \\
\text { kV com ou sem } \\
\text { RE-2 conectado } \\
\text { em Assis. }\end{array}$ \\
\hline EXECUTOR & PROCEDIMENTO/AÇÃO & $\begin{array}{l}\text { CONDIÇÃO/ } \\
\text { CONTROLE }\end{array}$ \\
\hline $\begin{array}{c}\text { Usina } \\
\text { Taquaruçu }\end{array}$ & $\begin{array}{l}\text {-Sincronizar } 1 \text { (uma) unidade } \\
\text { geradora e energizar o TR-7 } \\
440 / 138 \mathrm{kV} \text { assumindo carga } \\
\text { da região. } \\
\text { - Aguardar instruções do COS } \\
\text { para fechamento do paralelo } \\
\text { com a Usina Capivara. }\end{array}$ & $\begin{array}{l}\text {-Valor Máximo } \\
\text { de carga igual a } \\
80 \mathrm{MW}\end{array}$ \\
\hline EXECUTOR & PROCEDIMENTO/AÇÃO & $\begin{array}{l}\text { CONDIÇÃO/ } \\
\text { CONTROLE }\end{array}$ \\
\hline $\begin{array}{l}\text { Subestação } \\
\text { Assis }\end{array}$ & $\begin{array}{l}\text {-Conectar o RE-2 na LT Ca- } \\
\text { pivara / Assis. } \\
\text { •Recebendo tensão de Capi- } \\
\text { vara, aguardar } 1 \text { (um) minuto } \\
\text { para possibilitar ajustes de } \\
\text { tensão em Capivara e energi- } \\
\text { zar a barra-I, o TR-1 440/230 } \\
\text { kV e os TRs } 3 \text { e } 4230 / 88 \mathrm{kV} \\
\text { e assumir cargas da região. } \\
\text {-Após a confi rmação da } 3^{a} \\
\text { unidade geradora sincroni- } \\
\text { zada em Capivara, enviar ten- } \\
\text { são para Londrina. }\end{array}$ & $\begin{array}{l}\text {-Tensão } \leq 485 \\
\text { kV para energi- } \\
\text { zar o TR-1 } \\
\text { •Valor Máximo } \\
\text { de carga igual a } \\
65 \text { MW } \\
\text {-Tensão entre } \\
220 \text { e } 230 \mathrm{kV}\end{array}$ \\
\hline EXECUTOR & PROCEDIMENTO/AÇÃO & $\begin{array}{l}\text { CONDIÇÃO/ } \\
\text { CONTROLE }\end{array}$ \\
\hline $\begin{array}{c}\text { Subestação } \\
\text { Londrina }\end{array}$ & $\begin{array}{l}\bullet \text { Recebendo tensão de Assis, } \\
\text { normalizar a LI e restabelecer } \\
\text { carga da região. }\end{array}$ & $\begin{array}{l}\text { - Valor Máximo } \\
\text { de carga igual a } \\
80 \mathrm{MW}\end{array}$ \\
\hline
\end{tabular}

\section{GRAFOS CPM/PERT}

Os métodos CPM e PERT são baseados na identificação do caminho crítico de um processo, isto é, da seqüência das atividades que determinam a duração total do projeto. Para atingir esse objetivo, utilizam uma representação gráfica das relações entre as atividades do processo, na forma de um grafo orientado (denominado grafo CPM/PERT).

Assim, a abordagem de um problema (como a recomposição de sistemas elétricos) através de métodos de caminho crítico é realizada a partir da definição inicial da lista de ativida-
Tabela II - Exemplo de Lista de Atividades - Área Capivara

\begin{tabular}{|c|c|c|}
\hline Ativ. & Descrição & Preced. \\
\hline A & Capivara sincroniza 2 geradores & - \\
\hline $\mathrm{B}$ & Capivara energiza TR-5 440/138 kV & A \\
\hline $\mathrm{C}$ & $\begin{array}{l}\text { Capivara envia tensão para Taqua- } \\
\text { ruçu }\end{array}$ & B \\
\hline $\mathrm{D}$ & $\begin{array}{l}\text { Capivara confirma com Assis a cone- } \\
\text { xão do reator RE-2 }\end{array}$ & $\mathrm{C}$ \\
\hline E & Capivara envia tensão para Assis & $\mathrm{D}$ \\
\hline $\mathrm{F}$ & $\begin{array}{l}\text { Capivara sincroniza o terceiro gera- } \\
\text { dor }\end{array}$ & $\mathrm{E}$ \\
\hline $\mathrm{G}$ & $\begin{array}{l}\text { Capivara informa Assis que o ter- } \\
\text { ceiro gerador está sincronizado }\end{array}$ & $\mathrm{F}$ \\
\hline $\mathrm{H}$ & Taquaruçu sincroniza 1 gerador & - \\
\hline I & $\begin{array}{l}\text { Taquaruçu energiza TR-7 440/138 } \\
\mathrm{kV}\end{array}$ & $\mathrm{H}$ \\
\hline $\mathrm{J}$ & $\begin{array}{l}\text { COS notifica Taquaruçu que o para- } \\
\text { lelo com Capivara pode ser fechado }\end{array}$ & $\mathrm{C}$ \\
\hline K & $\begin{array}{l}\text { Taquaruçu fecha o paralelo com Ca- } \\
\text { pivara }\end{array}$ & I e J \\
\hline $\mathrm{L}$ & Assis conecta o reator RE-2 & - \\
\hline M & $\begin{array}{l}\text { Assis espera o ajuste de tensão em } \\
\text { Capivara }\end{array}$ & $\mathrm{E}$ e $\mathrm{L}$ \\
\hline $\mathrm{N}$ & $\begin{array}{l}\text { Assis energiza barra-I, TR-1 440/230 } \\
\mathrm{kV} \text { e TRs } 3 \text { e } 4 \text { 230/88 kV }\end{array}$ & M \\
\hline $\mathrm{O}$ & Assis envia tensão para Londrina & $\mathrm{Ge} \mathrm{N}$ \\
\hline $\mathrm{P}$ & $\begin{array}{l}\text { Londrina normaliza LI e restabelece } \\
\text { carga }\end{array}$ & $\mathrm{O}$ \\
\hline
\end{tabular}

des do processo sob análise. Definida a lista de atividades, o grafo CPM/PERT pode ser montado, para viabilizar a aplicação dos algoritmos de cálculo de custos e tempos envolvidos com o projeto de maneira eficiente. Nesse trabalho, são abordados os grafos do tipo "AoA" ("Activities-on-Arcs") ou redes orientadas por eventos, onde as atividades do processo são associadas às arestas do grafo CPM/PERT em construção. Os vértices do grafo são denominados etapas, que recebem usualmente uma identificação seqüencial. Essas etapas são os estágios do processo onde ocorre a convergência de atividades e, portanto, onde fica explícita a interdependência entre as mesmas. Nesse tipo de rede, pode existir apenas uma atividade entre cada par de etapas. Assim que o grafo CPM/PERT esteja disponível pode-se aplicar as técnicas de identificação de caminho crítico. 


\section{MODELOS ADOTADOS PARA AS ETA- PAS E ATIVIDADES DA RECOMPOSI- ÇÃO}

Foi adotada, aqui, uma abordagem orientada a objetos na especificação dos elementos do grafo CPM/PERT. A utilização de programação orientada a objetos para a definição de modelos de atividades e de etapas do grafo permite a adoção de uma representação física para os mesmos, mais próxima da sua natureza tecnológica/procedural. A despeito da maior utilização de memória computacional, essa abordagem permite que os elementos do grafo sejam instanciados de maneira independente, preservando características individuais das atividades. Além disso, torna fácil a extração de diferentes formas de representação matemática do grafo, como listas de arestas, listas de incidência ou matrizes de incidência.

\subsection{Modelo de Atividades}

As atividades são representadas, na rede "AoA", por arcos orientados do grafo CPM/PERT que se iniciam e terminam em etapas distintas. Esses arcos ficam relacionados a características como as diferentes estimativas de tempo de conclusão de cada atividade, recursos envolvidos e relações entre custo e tempo do processo. Utilizando uma abordagem baseada em objetos, o instanciamento das atividades de um plano pode ser diretamente realizado a partir da lista de atividades associada ao processo tecnológico em questão. A figura 2 apresenta o modelo genérico para atividades de um processo.

\subsection{Modelo de Etapas}

Os nós do grafo CPM/PERT representam as etapas do processo sob análise, e correspondem aos pontos temporais onde algumas atividades convergem para dar início a outras atividades. Neles, fica explícita, portanto, a dependência entre os diferentes componentes do processo. Elas também podem ser caracterizadas diretamente a partir da lista de atividades, de acordo com o modelo explicitado na figura 3.

\section{METODOLOGIA DE MONTAGEM AUTO- MÁTICA DOS GRAFOS CPM/PERT}

Uma vez definidos os modelos de objetos para as etapas e atividades, é necessário estabelecer um procedimento para satisfazer as relações de conectividade entre os elementos, caracterizando conseqüentemente a estrutura topológica do grafo CPM/PERT. Os passos que se seguem definem uma metodologia computacional, proposta por Mota et. al. (2004), que foi utilizada neste trabalho para realizar essa tarefa.

\begin{tabular}{|l|}
\hline Nome da Classe \\
Atividade CPM/PERT \\
\hline Campos (Variáveis) \\
Nó de origem: identificador da etapa inicial \\
Nó de destino: identificador da etapa final \\
t: tempo/recursos estimados para essa atividade \\
P: vetor de atividades precedentes \\
\hline Métodos mais Importantes (Funçôes) \\
Get/Set tempos(): retorna ou define os recursos/duração \\
Get/Set/Remove Origem/Destino(): retorna, define ou \\
remove as etapas inicial/final dessa atividade \\
Get/Set/Remove precedentes(): retorna, define ou remove \\
as atividades pré-requisito \\
\hline
\end{tabular}

Figura 2: Modelo de Atividades.

\begin{tabular}{|c|}
\hline $\begin{array}{l}\text { Nome da Classe } \\
\text { Etapa CPM/PERT }\end{array}$ \\
\hline $\begin{array}{l}\text { Campos (Variáveis) } \\
\text { Atividades de Entrada: conjunto de atividades que } \\
\text { terminam nessa etapa } \\
\text { Atividades de Saída: conjunto de atividades que } \\
\text { se iniciam nessa etapa }\end{array}$ \\
\hline $\begin{array}{l}\text { Métodos mais Importantes (Funções) } \\
\text { Get/Set/Remove entrada(): retorna, define ou remove } \\
\text { as atividades que terminam nessa etapa } \\
\text { Get/Set/Remove saída(): retorna, define ou remove } \\
\text { as atividades que se iniciam nessa etapa }\end{array}$ \\
\hline
\end{tabular}

Figura 3: Modelo de Etapas.

\section{Passo 1: Definição das arestas}

Neste passo, são definidas as arestas orientadas dos grafos CPM/PERT utilizando como entrada a estrutura da lista de atividades. Para cada componente dessa lista, são atribuídos os parâmetros de identificação e descrição de atividades precedentes. Este passo está representado pelo algoritmo a seguir.

\section{Seja $\boldsymbol{A}=$ conjunto de atividades}

Para cada linha da lista de atividades $\boldsymbol{i}$

$\boldsymbol{a}=$ nova atividade

Atribuir nome de a conforme a linha da lista de atividades Atribuir lista de pré-requisitos de a conforme a linha da lista de atividades

Adicionar a ao conjunto de atividades $\boldsymbol{A}$

\section{Passo 2: Definição das etapas de início das atividades}

Uma vez definidos os objetos associados às atividades, podese instanciar novos objetos, representando as etapas de início dos mesmos. Esses objetos do tipo nó do grafo são derivados pela inspeção das configurações de pré-requisitos definidas 
em cada objeto atividade, sendo-lhes atribuído um nome arbitrário e seqüencialmente numerado, conforme o algoritmo a seguir. Além disso, um teste para verificar a existência da etapa deve ser conduzido para cada atividade, uma vez que atividades diferentes podem se iniciar no mesmo nó.

Seja $N=$ conjunto de nós

$\boldsymbol{k}=0$;

Para cada atividade $\boldsymbol{i}$ definida em $\boldsymbol{A}$

$\boldsymbol{P}=$ conjunto de atividades pré-requisito de $\boldsymbol{i}$

Se não existe nó contendo exatamente todos os destinos de $\boldsymbol{P}$

$\boldsymbol{k}=\boldsymbol{k}+1$

$\boldsymbol{n}=$ novo nó que contém exatamente todos os destinos

de $\boldsymbol{P}$

nome de $\boldsymbol{n}=$ "Nó" $+\boldsymbol{k}$

Adicionar $\boldsymbol{n}$ ao conjunto de nós $\boldsymbol{N}$

$\boldsymbol{k}=\boldsymbol{k}+1$

$\boldsymbol{n}=$ novo nó que não contém nenhuma atividade

nome de $\boldsymbol{n}=$ "Start"

Adicionar $\boldsymbol{n}$ ao conjunto de nós $\boldsymbol{N}$

\section{Passo 3: Conexão das origens das atividades}

Uma vez que o conjunto inicial de nós é derivado a partir das listas de requisitos das próprias atividades, a conexão das origens das atividades às etapas já definidas é trivial. $\mathrm{O}$ algoritmo a seguir sintetiza as ações a serem tomadas nesse passo. É importante salientar que, computacionalmente, esse passo e o anterior podem ser realizados praticamente de maneira simultânea, embora, neste trabalho eles tenham sido explicitados separadamente com o propósito de proporcionar um melhor entendimento do processo.

\section{Para cada atividade $\boldsymbol{i}$ definida em $\boldsymbol{A}$}

$\boldsymbol{P}=$ conjunto de atividades pré-requisito de $\boldsymbol{i}$

Para cada nó $\boldsymbol{j}$ definido em $\mathbf{N}$

Se $\boldsymbol{j}$ contém exatamente todos os destinos de $\boldsymbol{P}$ então Faça a origem de i igual $a \boldsymbol{j}$

\section{Passo 4: Conexão dos possíveis destinos}

Nesse ponto do processo, as atividades já possuem suas origens conectadas a nós definidos, mas seus destinos ainda estão desconexos. Esse passo consiste em conectar os destinos, para cada atividade definida em $\mathbf{A}$, aos nós pertencentes ao conjunto $\mathbf{N}$ já definido, quando apenas uma etapa depende da conclusão da atividade em questão. Entretanto, é possível que mais de uma etapa dependa da conclusão de uma única atividade. Essas atividades são marcadas como possuindo dependência múltipla e tratadas nos passos que se seguem. $\mathrm{O}$ algoritmo a seguir sintetiza esse passo.

$\boldsymbol{n}=$ novo nó que não contém nenhuma atividade nome de $\boldsymbol{n}=$ "End"

Para cada atividade $\boldsymbol{i}$ definida em $\boldsymbol{A}$

$\boldsymbol{N D}=$ número de etapas dependentes de $\boldsymbol{i}=0$

Para cada nó $\boldsymbol{j}$ definido em $\mathbf{N}$

Se $\boldsymbol{j}$ contém o destino de $\boldsymbol{i}$

Marcar $\boldsymbol{j}$ como dependente de $\boldsymbol{i}$

$N D=N D+1$

Se $N D>1$

Marcar $\boldsymbol{i}$ como tendo dependência múltipla Senão

Se $N D=1$

Conectar destino de $\boldsymbol{i}$ ao nó dependente marcado $\boldsymbol{j}$

$\mathrm{Se} \mathbf{N D}=0$

Conectar destino de $\boldsymbol{i}$ ao nó final $\boldsymbol{n}$

Adicionar $\boldsymbol{n}$ ao conjunto de nós $\mathbf{N}$

\section{Passo 5: Criação de etapas de destino adicionais}

Nesse passo, representado no algoritmo que se segue, são atribuídas etapas de destino adicionais para as atividades com dependência múltipla. Essas etapas correspondem ao momento da conclusão da atividade em questão e são representadas por nós conectados ao destino das atividades que não puderam ser conectados no passo anterior. A criação de tais etapas é necessária para que o passo seguinte, onde a conectividade do grafo é concluída, possa ser executado.

Seja $\mathbf{N A}=$ conjunto de nós adicionais

Para cada atividade definida $\boldsymbol{i}$

Se i possui dependência múltipla

$\boldsymbol{n}=$ novo nó que contém $\boldsymbol{i}$

nome de $\boldsymbol{n}=$ "Nó adicional" + nome de $\boldsymbol{i}$

Adicionar n ao conjunto de nós $\mathbf{N A}$

\section{Passo 6: Criação de atividades-fantasma}

Para a execução desse passo, é necessário instanciar objetos atividade com duração e recursos nulos, ou seja, as atividades-fantasma (ou "dummies"). Nesse passo, as etapas que ainda não tem todos seus pré-requisitos atendidos sofrem uma busca conduzida pelas etapas adicionais criadas no passo anterior; quando a etapa de destino da atividade (ou atividades) pré-requisito é identificada, uma atividadefantasma é criada, iniciando-se no nó de destino adicional e terminando na etapa que iniciou a busca. O objetivo de uma atividade fantasma é, portanto, atender às restrições de precedência impostas pela lista de atividades e que não podem ser satisfeitas usando apenas o conjunto de atividades reais. $\mathrm{O}$ algoritmo a seguir representa esse passo.

Seja $\boldsymbol{A F}$ = conjunto de atividades-fantasma

Para cada nó adicional na definido em NA

$\boldsymbol{i}=$ atividade incidente em $\mathbf{n a}$

Para cada nó $\boldsymbol{j}$ definido em $\mathbf{N}$

Se $\boldsymbol{j}$ contém $\boldsymbol{i}$ então 
af = nova atividade fantasma com duração nula

Faça a origem de af igual à na

Faça o destino de af igual $a j$

Adicionar af ao conjunto de atividades-fantasma $\mathbf{A F}$

\section{Passo 7: Filtragem de atividades-fantasma desnecessárias}

Esse passo corresponde ao final do processo de atribuição das conexões do grafo CPM/PERT, onde as atividades-fantasma que não são relevantes são filtradas e descartadas da estrutura. A presença de atividades-fantasma desnecessárias pode ocorrer quando, para uma atividade com dependência múltipla, existe uma etapa que depende apenas da conclusão da atividade em questão. Nesse caso, essa etapa corresponde à conclusão individual da atividade e, portanto, coincide com a etapa adicional que foi criada para destino da atividade no passo 5. Embora esse passo tenha sido explicitado neste trabalho separadamente, para uma compreensão melhor do processo de construção do grafo, ele pode ser incorporado iterativamente aos passos 5 e 6 , visando a obtenção de uma maior eficiência computacional, assim como no caso do passo 3 . O algoritmo que se segue descreve esse passo.

Para cada atividade-fantasma af definida em $\boldsymbol{A F}$

$\boldsymbol{j}=$ nó de destino de af

Se $\boldsymbol{j}$ contém exatamente af então

$\boldsymbol{n}=$ nó de origem de $\boldsymbol{a f}$

transfira todas as atividades dependentes de $\boldsymbol{j}$ para $\boldsymbol{n}$

elimine $\boldsymbol{j}$

atualize $\mathbf{N}$

elimine af

atualize $\boldsymbol{A F}$

\section{EXEMPLOS DE APLICAÇÃO}

\subsection{Aplicação em um Processo Genérico}

Pelo fato de agregar uma maior flexibilidade na sua formulação, decorrente da abordagem orientada a objetos, a metodologia proposta pode ser utilizada para modelar diferentes processos tecnológicos. Para verificar essa característica, a técnica de montagem automática de grafos CPM/PERT foi aplicada a um exemplo extraído de Goldbarg (2000), correspondente ao processo de montagem de uma cabine de simulação, cuja lista de atividades é reproduzida na figura 4.

A aplicação dos passo 1-7 do método de montagem automática gerou os resultados descritos a seguir.

\section{Passo 1: Instanciamento das arestas}

As arestas são instanciadas diretamente por inspeção da lista de atividades, resultando em nove objetos, conforme a figura 5 .

\begin{tabular}{|l|c|c|}
\hline \multicolumn{1}{|c|}{ Atividade } & $\begin{array}{c}\text { Atividades } \\
\text { Precedentes }\end{array}$ & Duração \\
\hline A: Projeto de Simulação & $\cdots$ & 2 \\
\hline B: Treinamento de pessoal & A & 9 \\
\hline C: Construção de Instalações Especiais & A & 4 \\
\hline D: Certificação de Instalações & C & 1 \\
\hline E: Aquisição de Material & A & 1 \\
\hline F: Aferição dos Instrumentos & E & 2 \\
\hline G: Teste do material & D,F & 3 \\
\hline H: Montagem da Cabine de simulação & B, G & 1 \\
\hline I: Execução da Simulação & H & 2 \\
\hline
\end{tabular}

Figura 4: Lista de atividades de um processo de montagem de uma cabine de simulação.

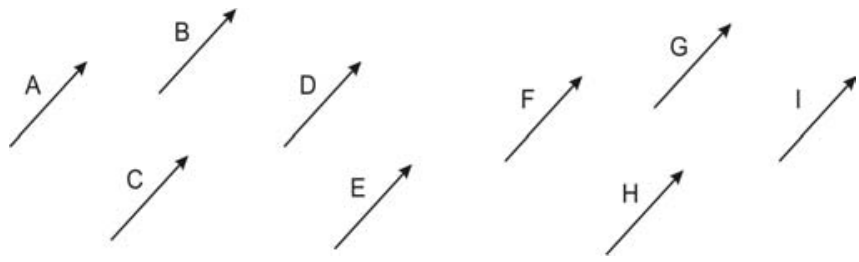

Figura 5: Arestas instanciadas.

\section{Passo 2: Instanciamento das etapas de início}

Assim como no caso das arestas, as etapas também são instanciadas diretamente por inspeção da lista de atividades, resultando em sete objetos (seis etapas extraídas da lista e mais uma instanciada como nó de início do processo), conforme ilustrado na figura 6 .

\section{Passo 3: Conexão das origens}

De posse da lista de arestas e da lista de vértices, os inícios das atividades são conectados às etapas correspondentes, conforme ilustrado na figura 7 .

\section{Passo 4: Conexão dos possíveis destinos}

A primeira ação nesse passo é o instanciamento de uma atividade adicional para representar o nó final do processo (nesse caso, instanciado como o vértice número 7). A seguir, os destinos das atividades são conectados ao conjunto de nós disponível (quando possível), resultando no grafo ilustrado na figura 8 .

Os passos 5 (instanciamento de destinos adicionais), 6 (instanciamento de atividades-fantasma) e 7 (filtragem de atividades-fantasma) não são necessários nesse exemplo, 


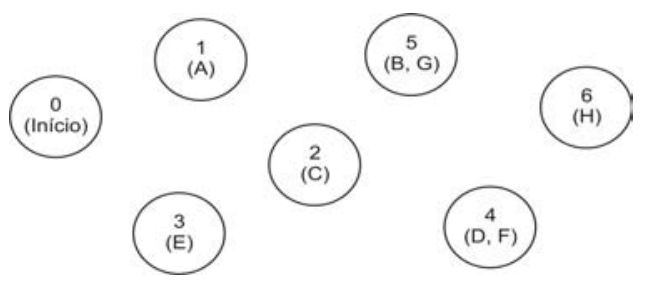

Figura 6: Etapas de início.



Figura 7: Conexão das atividades aos inícios.

uma vez que todas as atividades já possuem suas etapas inicial e final definidas.

\subsection{Aplicação em um Plano de Recompo- sição}

Para ilustrar a aplicação dessa metodologia de montagem automática de redes à modelagem de planos de recomposição do SIN, a seqüência de sete passos do algoritmo de montagem automática foi aplicada à lista de atividades associada à instrução operativa da região Capivara, explicitada previamente na tabela II.

Observando essa lista de atividades, é possível explorar as relações de precedência entre as diferentes ações que compõem o plano para determinar, então, a estrutura topológica do grafo de caminho crítico correspondente à IO de recomposição da área Capivara. A figura 9 representa o grafo CPM/PERT obtido para esse caso.

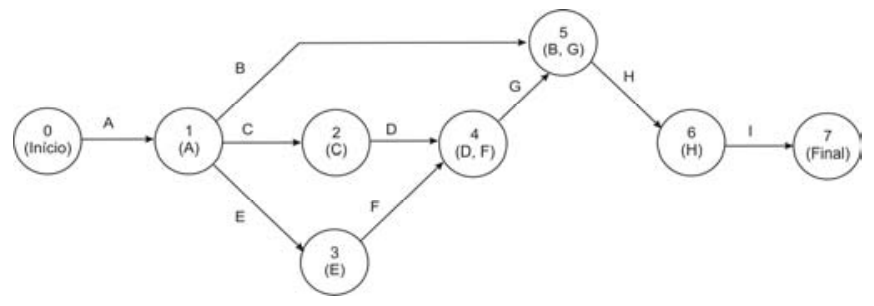

Figura 8: Grafo montado para um processo de montagem de uma cabine de simulação.

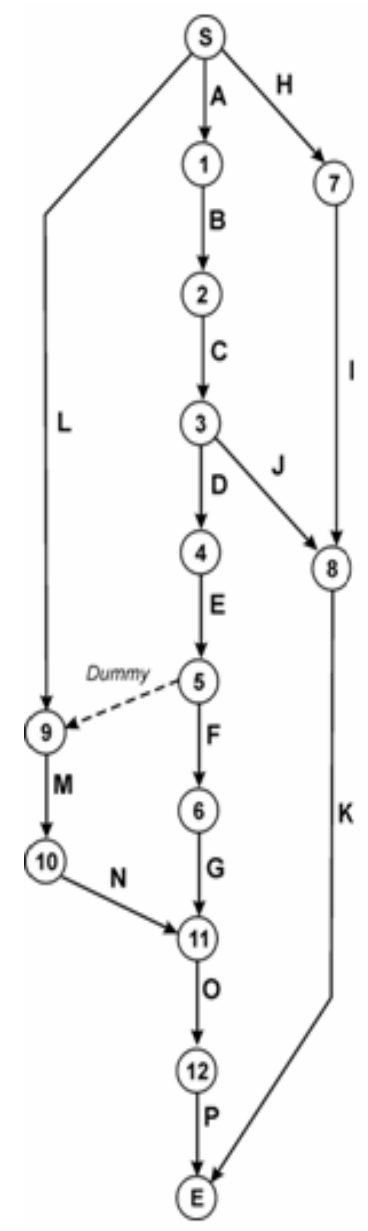

Figura 9: Grafo CPM/PERT para a recomposição da área Capivara.

\subsection{Observações Adicionais}

Com o grafo CPM/PERT de uma IO disponível, é possível conduzir uma série de análises que fornecem informações relevantes acerca do processo de recomposição em questão. Os procedimentos envolvidos em tais análises estão descritos em maior detalhe em Mota et. al. (2005) e em Moder (1970). Como exemplo, a tabela III apresenta os tempos e respectivos valores de desvio padrão, estimados para a conclusão das atividades de recomposição da área Capivara, representadas anteriormente na tabela II.

Os resultados do processamento dessa estrutura com os métodos convencionais de caminho crítico e com as técnicas citadas, forneceram um tempo total estimado para recomposição dessa área igual a 93,5 unidades de tempo, com 9 atividades críticas $(A, B, C, D, E, F, G, O$ e $P)$ e um custo estimado para interrupção de aproximadamente 650.000 dólares, considerando-se valores de custo unitário de interrupção por segmento de consumo descritos por Gomes et. al. (1997). 
Tabela III - Estimativas dos tempos de conclusão das atividades de recomposição da área Capivara

\begin{tabular}{|c|c|c||c|c|c|}
\hline Ativ. & $\begin{array}{c}\mathbf{t}_{m} \\
(\text { u.t. })\end{array}$ & $\begin{array}{c}\sigma_{e} \\
\text { (u.t.) }\end{array}$ & Ativ. & $\begin{array}{c}\mathbf{t}_{m} \\
(\text { u.t. })\end{array}$ & $\begin{array}{c}\sigma_{e} \\
(\text { u.t. })\end{array}$ \\
\hline $\mathbf{A}$ & 40 & 8,33 & $\mathbf{I}$ & 5 & 1,17 \\
\hline $\mathbf{B}$ & 5 & 1,17 & $\mathbf{J}$ & 1 & 0,41 \\
\hline $\mathbf{C}$ & 5 & 0,83 & $\mathbf{K}$ & 5 & 0,5 \\
\hline $\mathbf{D}$ & 1 & 0,25 & $\mathbf{L}$ & 5 & 1 \\
\hline $\mathbf{E}$ & 5 & 0,83 & $\mathbf{M}$ & 1 & 0,25 \\
\hline $\mathbf{F}$ & 20 & 3,33 & $\mathbf{N}$ & 15 & 0,5 \\
\hline $\mathbf{G}$ & 1 & 0,25 & $\mathbf{O}$ & 5 & 0,83 \\
\hline $\mathbf{H}$ & 40 & 5 & $\mathbf{P}$ & 5 & 1,33 \\
\hline
\end{tabular}

A execução dessas tarefas tomou menos que um segundo, em um microcomputador pessoal, para processar desde a aquisição das informações acerca da lista de atividades (disponibilizada em arquivos tipo texto), passando pela geração do grafo de caminho crítico e terminando com a execução das funções de análise de tempos e custos associados ao grafo CPM/PERT.

É importante salientar que essa estrutura conexa de interdependência entre as ações de recomposição (o grafo CPM/PERT) pode ser aproveitada para a construção de representações gráficas em interfaces de usuário, permitindo que os operadores acompanhem, visualmente, os procedimentos de reenergização, durante treinamentos e no uso de programas de apoio à recomposição.

Finalmente, pode-se destacar que se o sistema sob análise fosse de maior porte (maior número de barras e linhas) ou se a solução do problema estivesse mais distante eletricamente do local sob recomposição no início do grafo, o modelo resultante da aplicação da metodologia teria um maior número de nós e arcos, uma vez que o plano de recomposição correspondente teria um maior número de ações de recomposição; os procedimentos para modelagem, entretanto, podem ser aplicados exatamente da mesma maneira, fornecendo resultados com esforço computacional proporcionalmente maior (Mota \& Mota, 2006).

\section{CONCLUSÕES}

Nesse trabalho, é explorado o modelo baseado em grafos dirigidos, do tipo CPM/PERT, para representar as seqüências de ações de reenergização de sistemas elétricos de potência após blecautes. Entretanto, é fundamental destacar que a operação de redes de energia no seu estado restaurativo envolve dois aspectos que tornam essa modelagem uma tarefa árdua, quando se considera a construção manual dos diagra- mas de caminho crítico:

(a) o planejamento da recomposição necessita da análise de um grande número de opções de reenergização para um mesmo cenário de interrupção e, assim, requer que um grande número de diferentes listas de atividades seja analisado;

(b) na utilização das instruções operativas como apoio à recomposição em tempo real, pode ser necessária a alteração de algumas das atividades que compõem o plano em decorrência de imprevistos na operação e, portanto, o diagrama do processo deve ser atualizado, o que, manualmente, é extremamente difícil nessa situação.

Nesse sentido, a metodologia de modelagem automática dos planos, conforme exposta previamente, permite o armazenamento das informações contidas nos planos de recomposição em estruturas matemáticas e computacionais bem definidas. Essas estruturas podem ser exploradas para fornecer um grande número de informações sobre os planos de recomposição, relacionadas a sua duração, custos e decisões sobre investimentos (Moder, 1970; Mota, 2005; Sivazlian, 1975) em um tempo bastante reduzido, o que vem ao encontro das necessidades específicas do problema de restabelecimento do serviço após distúrbios de grandes proporções.

\section{AGRADECIMENTOS}

Os autores gostariam de agradecer à Fundação de Amparo a Pesquisa do Estado de São Paulo (FAPESP) pelo suporte durante o desenvolvimento deste trabalho.

\section{REFERÊNCIAS}

Adibi, M.M. (2000). Power System Restoration - Methodologies and Implementation Strategies, New York: IEEE Press.

Adibi, M.M., \& D.P. Milanicz (1999). Estimating Restoration Duration, IEEE Transactions on Power Systems, vol. 14 , n. 4 , Nov.

Goldbarg, M.C., \& H.P.L. Luna (2000). Otimização Combinatória e Programação Linear-Modelos e Algoritmos, Rio de Janeiro: Editora Campus.

Gomes, P. \& M. Th. Schilling (1997). Custo de Interrupção: Conceituação, Metodologia de Avaliação, Valores Existentes e Aplicações, Anais do XIV SNPTEE - Seminário Nacional de Produção e Transmissão de Energia Elétrica, Belém (PA).

Moder, J.J. \& C.R. Phillips (1970). Project Management with CPM and PERT, New York: Van Nostrand Reinhold Company. 
Mota, A.A., L.T.M. Mota e A. Morelato (2004). Metodologia Orientada a Objetos para a Construção Automática de Grafos CPM/PERT, Anais do XXXVI SBPO - Simpósio Brasileiro de Pesquisa Operacional, São João Del Rei (MG).

Mota, A.A., L.T.M. Mota e A. Morelato (2005). Avaliação da Qualidade de Planos de Recomposição de Sistemas de Energia Elétrica, Anais do XVIII SNPTEE - Seminário Nacional de Produção e Transmissão de Energia Elétrica, Curitiba (PR).

Mota, A.A. \& L.T.M. Mota (2006). Análise dos Tempos de Recomposição das Áreas Capivara e Marimbondo. Relatório 02/2006, Operador Nacional do Sistema Elétrico - ONS, Brasília (DF).

Morelato, A. \& A. Monticelli (1989). Heuristic Search Approach to Distribution System Restoration", IEEE Transactions on Power Delivery, vol. 4, n.4.

ONS - Operador Nacional do Sistema Elétrico (2004). Procedimentos de Rede: Módulo 10, Centro Nacional de Operação do Sistema - CNOS, Brasília (DF).

Sivazlian, B.D. \& L.E. Stanfel (1975). Optimization Techniques in Operations Research, New Jersey: PrenticeHall. 